University of Wollongong

Research Online

Faculty of Social Sciences - Papers (Archive) Faculty of Arts, Social Sciences \& Humanities

2002

Can the Q Link Ally R, a form of Sympathetic Resonance Technology (SRTTM), attenuate acute mobile phone-related changes to neural function?

Rodney J. Croft

University of Wollongong, rcroft@uow.edu.au

Jody Chandler

University of Wollongong

Adrian P. Burgess

Imperial College School of Medicine

Robert Barry

University of Wollongong, rbarry@uow.edu.au

John D. Williams

Coventry University

See next page for additional authors

Follow this and additional works at: https://ro.uow.edu.au/sspapers

Part of the Education Commons, and the Social and Behavioral Sciences Commons

Research Online is the open access institutional repository for the University of Wollongong. For further information contact the UOW Library: research-pubs@uow.edu.au 


\title{
Can the Q Link Ally R, a form of Sympathetic Resonance Technology (SRTTM), attenuate acute mobile phone-related changes to neural function?
}

\begin{abstract}
Objectives: Exposure to active mobile phones (MP) has been shown to affect human neural function as shown by the electroencephalogram (EEG). Although it has not been determined whether such effects are harmful, a number of devices have been developed that attempt to minimize these MP-related effects.

One such device, the Q Link Ally ${ }^{\circledR}$ (QL; Clarus Products, International, L.L.C., San Rafael, CA), is argued to affect the human organism in such a way as to attenuate the effect of MPs. The present pilot study was designed to determine whether there is any indication that QL does alter MP-related effects on the human EEG.

Design: Twenty-four (24) subjects participated in a single-blind, fully counterbalanced crossover design in which subjects' resting EEG and phase-locked neural responses to auditory stimuli were assessed under conditions of either active MP or active MP plus QL.

Results: The addition of QL to the MP condition increased resting EEG in the gamma range and did so as a function of exposure duration, and it attenuated MP-related effects in the delta and alpha range (at trend-level). The addition of the QL also affected phase-locked neural responses, with a laterality reversal in the alpha range and an alteration to changes over time in the delta range, a reduction of the MP-related beta decrease over time at fronto-posterior sites, and a global reduction in the gamma range that increased as a function of exposure duration. No unambiguous relations were found between these changes and either performance or psychologic state.
\end{abstract}

Conclusions: This pilot study suggests that the addition of the QL to active MP-exposure does affect neural function in humans, altering both resting EEG patterns and the evoked neural response to auditory stimuli, and that there is a tendency for some MP-related changes to the EEG to be attenuated by the QL.

\section{Keywords}

changes, related, mobile, attenuate, srttm, can, resonance, sympathetic, ally, link, q, r, neural, phone, acute, function, technology, form

\section{Disciplines}

Education | Social and Behavioral Sciences

\section{Publication Details}

Croft, R. J., Chandler, J. S., Burgess, A. P., Barry, R. J., Williams, J. D. \& Clarke, A. R. (2002). Can the Q Link Ally ${ }^{R}$, a form of Sympathetic Resonance Technology $\left(\mathrm{SRT}^{\mathrm{TM}}{ }^{\mathrm{M}}\right.$ ), attenuate acute mobile phone-related changes to neural function?. The Journal of Alternative and Complementary Medicine, 8 (4), 427-435.

\section{Authors}

Rodney J. Croft, Jody Chandler, Adrian P. Burgess, Robert Barry, John D. Williams, and Adam Clarke 


\title{
Can the Q Link Ally, ${ }^{\circledR}$ a Form of Sympathetic Resonance Technology (SRT $\left.{ }^{\mathrm{TM}}\right)$, Attenuate Acute Mobile Phone-Related Changes to Neural Function?
}

\author{
RODNEY J. CROFT, Ph.D. ${ }^{1,2}$ JODY S. CHANDLER, B.Sc., ${ }^{1}$ ADRIAN P. BURGESS, Ph.D., ${ }^{3}$ \\ ROBERT J. BARRY, Ph.D., ${ }^{1}$ JOHN D. WILLIAMS, Ph.D., ${ }^{4}$ and ADAM R. CLARKE, Ph.D. ${ }^{1}$
}

\begin{abstract}
Objectives: Exposure to active mobile phones (MP) has been shown to affect human neural function as shown by the electroencephalogram (EEG). Although it has not been determined whether such effects are harmful, a number of devices have been developed that attempt to minimize these MP-related effects. One such device, the Q Link Ally ${ }^{\circledR}$ (QL; Clarus Products, International, L.L.C., San Rafael, CA), is argued to affect the human organism in such a way as to attenuate the effect of MPs. The present pilot study was designed to determine whether there is any indication that QL does alter MP-related effects on the human EEG.

Design: Twenty-four (24) subjects participated in a single-blind, fully counterbalanced crossover design in which subjects' resting EEG and phase-locked neural responses to auditory stimuli were assessed under conditions of either active MP or active MP plus QL.

Results: The addition of QL to the MP condition increased resting EEG in the gamma range and did so as a function of exposure duration, and it attenuated MP-related effects in the delta and alpha range (at trend-level). The addition of the QL also affected phase-locked neural responses, with a laterality reversal in the alpha range and an alteration to changes over time in the delta range, a reduction of the MP-related beta decrease over time at fronto-posterior sites, and a global reduction in the gamma range that increased as a function of exposure duration. No unambiguous relations were found between these changes and either performance or psychologic state.

Conclusions: This pilot study suggests that the addition of the QL to active MP-exposure does affect neural function in humans, altering both resting EEG patterns and the evoked neural response to auditory stimuli, and that there is a tendency for some MP-related changes to the EEG to be attenuated by the QL.
\end{abstract}

\section{INTRODUCTION}

$\mathbf{M}$ obile phones (MP) typically operate via a 900-1800 MHz pulsed-signal the output of which can be as high as $250 \mathrm{~mW}$. There is concern that exposure to this signal may affect humans, with a resultant body of research testing for MP related changes in human physiology and pathology. Research has failed to find consistent relations between MP use and human pathology, however, in terms of neural function there is evidence that MPs have a $\mathrm{cu}^{-}$

\footnotetext{
${ }^{1}$ Brain and Behaviour Research Institute, University of Wollongong, Wollongong, Australia.

${ }^{2}$ Centre for Neuropsychopharmacology, Swinburne University of Technology, Hawthorn, Australia.

${ }^{3}$ Department of Cognitive Neuroscience and Behaviour, Imperial College Medical School, London United Kingdom.

${ }^{4}$ Department of Psychology, Coventry University, Coventry United Kingdom.
} 
mulative effect on neural function (as indexed by the electroencephalogram [EEG]), becoming pronounced after 15 to 20 minutes of exposure (Reiser et al., 1995; Lebedeva et al., 2000; Croft et al., 2002). For example, we recently demonstrated that MPs alter both resting EEG and the phase-locked neural response to auditory stimuli as a function of exposure duration, with these changes related to changes in both psychological state and reaction time measures (Croft et al., 2002). Although it has not been shown that such changes are detrimental to the user, a number of methods have been developed that purport to attenuate the effect of MPs. The present paper reports on a pilot study designed to test one such device, the $\mathrm{Q}$ Link Ally (QL; Clarus Products International, L.L.C., San Rafael, CA), in attenuating the effects of an activated MP on human EEG.

The QL is a "black box" portable electromagnetic field (EMF)-emitting device that emits low-dose (100 $\mathrm{mW}$ power uptake) continuous waves at $7377 \mathrm{MHz}$ (plus a series of upper harmonics), and is powered by a $9 \mathrm{~V}$ D.C. source. It is argued by the developer that this EMF acts as a carrier wave for subatomic "information," and that this information assists in strengthening an organism's resilience to stressors. However, there are a number of elements to the above theory that are not verifiable (some because critical details have not been made available by the developer, and others because science does not have the requisite tools at present). Thus, this study tests an aspect of this thesis that is within the scope of electrophysiology-whether it alters the MPrelated effect on neural function (in terms of the EEG). In order to perform this test, neural function while exposed to an active MP was compared to neural function while exposed to both an active MP and QL. The measures used were resting EEG (the subject sits relaxed with their eyes open), the early phase-locked neural response to a number of tones (the subject selectively responds to one of two tones differing only in frequency), performance (reaction time), and psychologic state (how "activated" the subject is). The null hypothesis being tested is that the QL has no effect on any of the measures (i.e., for each measure, "MP" = "QL + $\left.M P^{\prime \prime}\right)$. This study forms part of a larger research project designed to determine the effect of MPs on human EEGs, with the "MP versus Control" comparisons reported elsewhere (Croft et al., 2002).

\section{MATERIALS AND METHODS}

\section{Subjects}

Sixteen (16) males and 8 females participated in the study and were paid $\$ 5$; ages ranged from 19 to 48 years (mean, 27.5) and 20 were righthanded. Subjects reported no hearing deficits or neurologic histories, gave written informed consent, and were free to withdraw from the study at any time without penalty. The study was approved by the local ethics committee.

\section{Procedure}

On arrival at the laboratory, subjects completed an informed consent form followed by demographics and personality questionnaires. They were fitted with EEG recording apparatus and seated in a sound attenuated recording booth where an EMF stressor (EMF) and an EMF attenuator $\left(\mathrm{EMF}_{\mathrm{att}}\right)$ were attached. Subjects then performed a 3-minute auditory discrimination task after which the subject sat resting for 2 minutes with their eyes open (Resting EEG). This 5-minute EEG protocol was repeated four times, and this 20-minute series of tasks was performed three times, each with a different background condition (resulting in a total EEG recording time of 60 minutes). The three background conditions were EMF, consisting of exposure to an active MP, EMF att, consisting of concurrent exposure to an active MP and QL, and CONTROL, consisting of neither MP nor $\mathrm{EMF}_{\mathrm{att}}$. Condition order was counterbalanced across subjects, and subjects were unaware of which condition they were engaged in (single-blind). In addition to this, immediately before and after each 20-minute series of tasks, the Thaya Activation-Deactivation Adjective Check List (AD-ACL; Thaya, 1967) was administered. Subjects were continuously monitored throughout the experiment via closed-circuit television.

\section{Discrimination task}

Each discrimination task consisted of 9070 $\mathrm{dB}$ binaural tones, of which half were $1100 \mathrm{~Hz}$ 
[High] and half were $1000 \mathrm{~Hz}$ [Low]. Tone duration was $60 \mathrm{~ms}$, with a variable interstimulus interval of $2 \pm 1 \mathrm{~s}$, and stimuli were presented through speakers positioned approximately 1.5 $\mathrm{m}$ in front of the subject. Subjects were asked to respond as quickly and accurately as possible to the target tones with a button press. Target-frequency and response-hand were constant within subject and counterbalanced and randomly assigned between subjects. A trial block was given prior to the testing session to familiarize subjects with the task. Behavioral measures obtained were accuracy and reaction time, and to encourage peak performance throughout the session, after the practice block subjects were offered monetary reward $(\$ 10)$ to perform at least as accurately and fast during the remainder of the session as they did in the practice block.

\section{Materials}

To enhance ecological validity, a standard Nokia 5110 mobile phone (Nokia Australia, Sydney, New South Wales, Australia) was used as a receiver to generate EMF $(900 \mathrm{MHz} \mathrm{EMF}$, $217 \mathrm{~Hz}$ pulse rate; $0.577 \mu$ s pulse width; estimated maximum power 3-4 $\mathrm{mW}$; actual emissions during experiment not measured). This was positioned $5-\mathrm{cm}$ radial to the subject's scalp midway between $\mathrm{Oz}$ and $\mathrm{Pz}$, using a nonmetallic external bracket. To reduce variability caused by changes in signal strength, a standard script was used that was sent by a phone in another room. The standard script consisted of spoken extracts from a radio play. These were not audible to the subject, but were played during each condition. The QL was powered by $240 \mathrm{~V}$ mains with a 9-V DC transformer, and was positioned over the subject's chest (left sternum) using a comfortable cheststrap and operated from a separate room. The position of EMF and $\mathrm{EMF}_{\text {att }}$ were constant throughout the experiment.

\section{Data acquisition and analysis}

Behavioral and psychologic measures. Accuracy and reaction time (RT) indices were derived from the discrimination task as the percentage of total targets responded to and the mean time taken to respond to these targets respectively. The first four stimuli from each 3-minute task were excluded from the above measures to reduce variability because of confounding effects related to task recommencement. The AD-ACL (Thaya, 1967) consists of 20 words that describe mood or feelings. Subjects rate the degree to which these adjectives describe their mood at that particular point in time on a four-point Likert-scale. Items relate to the adjectives "calm," "excited," "tired," and "tense," and average to form an "activation" scale (low scores represent high activation levels). A difference score was computed from this scale (i.e., "condition minus preexperiment score") and used as a dependent measure (this is referred to as "Activation").

Electrophysiologic measures. EEG data were collected from 19 scalp sites (Fp1, Fp2, F7, F3, Fz, F4, F8, T3, C3, Cz, C4, T4, T5, P3, Pz, P4, T6, $\mathrm{O} 1, \mathrm{O} 2)$ using an electrode cap with tin electrodes and referenced to linked ears (AMLABII acquisition system; AMLAB Technologies, Sydney, New South Wales, Australia). Electrooculographic data were recorded using tin electrodes above and below the left eye, and on the outer canthus of each eye. Data were continuously sampled at $512 \mathrm{~Hz}$ with a 0.05 to $120 \mathrm{~Hz}$ bandpass. Impedances were kept below 5 $\mathrm{kOhm}$. Data were grouped into the following scalp regions: front left $(\mathrm{FL})=$ mean $[\mathrm{FP} 1, \mathrm{~F} 3$, F7]; front midline $(\mathrm{FM})=\mathrm{Fz}$; front right $(\mathrm{FR})=$ mean $[\mathrm{FP} 2, \mathrm{~F} 4, \mathrm{~F} 8]$; centre left $(\mathrm{CL})=$ mean $[\mathrm{C} 3$, $\mathrm{T} 3$; ; centre midline $(\mathrm{CM})=\mathrm{Cz}$; center right $(\mathrm{CR})=$ mean $[\mathrm{C} 4, \mathrm{~T} 4]$; posterior left $(\mathrm{PL})=$ mean [T5, P3, O1]; posterior midline $(\mathrm{PM})=$ $\mathrm{Pz}$; posterior right $(\mathrm{PR})=$ mean $[\mathrm{T} 6, \mathrm{P} 4, \mathrm{O} 2]$.

For each 2-minute resting EEG period, the 30-90-second period was EOG-corrected (Semlitsch et al., 1986), divided into 2 -second bins, Fast Fourier transformed ( $10 \%$ cosine window) and averaged. Dependent variables were resultant EEG amplitude values grouped into five frequency bands: 1 to $4 \mathrm{~Hz}$ (delta); 4 to $8 \mathrm{~Hz}$ (theta); 8 to $12 \mathrm{~Hz}$ (alpha); 12 to $30 \mathrm{~Hz}$ (beta); 30 to 45 $\mathrm{Hz}$ (gamma). These are referred to as "FFT."

For each 3-minute discrimination task, data were EOG-corrected (Semlitsch et al., 1986), epoched -1500 to $1000 \mathrm{~ms}$ poststimulus, and the phase-locked component (mean) of these epochs computed for the target and nontarget stimuli separately, for each of the frequency bands defined above for FFT data (12 dB/octave roll-off; zero phase shift). Dependent mea- 
sures for this task were peak percentage power change (relative to the -1000 to $-500 \mathrm{~ms}$ baseline) in the early sensory phase-locked response (0-200 ms poststimulus) for each of the frequency bands (early sensory change; ESC).

\section{Statistical analyses}

Results for the MP versus CONTROL comparison have been reported elsewhere (Croft et al., 2002) and will not be addressed here. However, to allow the reader to compare those results to this study, relevant means and standard deviations are given in Figure 1, along with a brief description of the MP effect. For each of the FFT, ESC, Activation, and RT measures, orthogonal repeated measures polynomial contrasts were used to test for relations between these indices and condition (QL + MP versus $\mathrm{MP}$ ), and where appropriate, time (1st versus 2nd half of each 20-minute condition) and scalp location (sagittal [frontal, central, posterior]; lateral [left, midline, right]). As each condition occurred 1st, 2nd and 3rd an equal number of times, for each dependent variable "order" was treated as a noise variable and removed by converting scores within each order (and where appropriate frequency) to $z$ scores (after being transformed to normality where appropriate). To maintain appropriate alpha levels, for each of the above indices, the number of planned contrasts was restricted to the number of error degrees of freedom (Tabacknick and Fidell, 1989). Only effects caused by condition or interactions between condition and topography, time or type are reported here.

To determine if any QL-related EEG changes were related to subjective state or performance measures, for the QL + MP condition, correlations were performed between the sagittal, lateral, and temporal dimensions of any significant contrasts, and both RT and Activation. For example, for a condition* laterality* (left versus right) effect, a variable would be computed (mean left-mean right hemisphere sites) and correlations performed between this new variable and both RT and Activation for the QL +

*The asterisk in this and similar phrasing, throughout this paper means "by" (e.g., no condition or condition by time effects....).
MP condition data. Nonparametric correlations were used because of difficulties normalizing data, and as two tests were performed per significant contrast, $\alpha$ was reduced to 0.025 .

To facilitate understanding of the effect of the QL, a summary of the results from Croft et al. 2002 follows. No differences were found between the mobile phone and control condition on reaction time $(p>0.45)$ or psychologic activation (THAYA; $p=0.08$ ). In terms of resting EEG, while there was less delta power over right hemisphere sites in the control condition, this differential was accentuated in the MP condition $(p=0.041)$, and while there was no affect of MP exposure on alpha at frontal sites, there was an enhancement of alpha over posterior sites in the EMF condition $(p=0.017)$. Further, whereas alpha did not change over time in the CONTROL condition, it increased over time in the MP condition at midline sites $(p=0.028)$. In relation to the phase-locked neural responses, whereas the early sensory theta response decreased over time primarily at midline sites in the CONTROL condition, this decrement was attenuated in the MP condition $(p=0.038)$. Exposure to the active MP also caused a global reduction in the early beta response $(p=0.032)$, with this reduction the result of an enhancement of the natural beta reduction over time that was greatest at frontal and posterior sites $(p=0.035)$. Whereas there was no effect of the active mobile phone exposure at lateral frontal or midline posterior sites, it increased the gamma response at midline frontal and lateral posterior sites $(p=0.021)$.

\section{RESULTS}

\section{Behavioral and psychologic measures}

Subjects' mean accuracy levels were 99.3\% (standard deviation $[\mathrm{SD}]=1.3)$ and $99.2 \%(\mathrm{SD}=$ $1.4)$, and their RTs $383.4 \mathrm{~ms}(\mathrm{SD}=75.4)$ and 381.5 $\mathrm{ms}(\mathrm{SD}=85.4)$ for the MP and QL+MP conditions, respectively. To approximate normality, reaction time data were transformed according to "t_RT = natural log (RT)." There were no condition or condition * time effects for RT $(F[1,23]<$ $1.07 ; p>0.313)$, and no condition effect for Activation $(F[1,23]=0.64 ; 0.432)$. 


\section{Electrophysiologic measures}

To approximate normality, data were transformed according to "t_FFT = natural log (FFT)." Whereas there was a MP-related decrease in delta at right relative to left hemisphere sites, there was a trend-level normalization of this pattern with the addition of the QL $(F[1,23]=3.37 ; p=0.079$; condition * lateral [left versus right]; Fig. 1A). This normalization did not relate to Activation or RT changes $(r[24]<0.26 ; p>0.264)$. The MP-related enhancement of alpha over posterior sites was not altered by the QL $(F[1,23]=0.25 ; p=0.620$; condition * sagittal [frontal versus posterior]; Fig. 1B), however, the midline alpha increase over time in the MP condition was affected by the QL, with a trend-level reduction over time $(F[1,23]=2.95 ; p=0.099 ;$ condition * lateral [lateral versus midline] ${ }^{*}$ time; Fig. $1 C$ ). This reduction was related to increased Activation (trend-level; $r[24]=-0.42 ; p=0.052$ ) but not RT $(r[24]=0.05 ; p=0.824)$. The addition of the $\mathrm{QL}$ increased midline gamma $(\mathrm{F}[1,23]=4.62$; $p=0.042$; condition * laterality [lateral versus midline]), and this increase was inversely related to Activation (trend-level; $r$ [24] = $-0.44, p=0.03)$ but not to RT $(r[24]=0.22$; $p=0.304)$. This midline gamma increase was largest at fronto-posterior sites $(F[1,23]=5.49$; $p=0.028$; condition * lateral [lateral versus midline] * sagittal [central versus fronto-posterior]; Fig. 2A) and this particular pattern was not related to Activation or RT $(r[24]<0.26 ; p\rangle$ 0.218 ). Gamma increased as a function of exposure duration across the scalp in QL + MP, whereas the MP condition resulted in a reduction at posterior sites $(F[1,23]=4.62 ; p=0.042$; condition * sagittal [frontal versus posterior] * time; Fig. 2B), but this difference did not relate to Activation or RT $(r[24]<0.02 ; p>0.910)$.

Discrimination task. To approximate normality, data were transformed according to "t_ESC = natural $\log$ (100-ESC)," for which more positive t_ESC values represent larger neural responses. The addition of the QL attenuated the left hemisphere decrease and enhanced the right hemisphere decrease in evoked delta over time (relative to the MP condition; $F[1,23]=14.09 ; p<0.001$; condition * lateral [left versus right] * time; Fig. 2C). This lat- eralization pattern was inversely related to RT (trend-level; $r[24]=-0.41 ; p=0.048$ ), but not related to Activation $(r[24]=0.16 ; p=0.445)$. The MP-related attenuation of lateral (relative to midline) theta over time was not significantly affected by the addition of the QL $(F[1,23]=1.63$; $p=0.214$; condition * laterality [lateral versus midline] * time; Fig. 1D). The addition of the QL increased left and decreased right hemisphere alpha $(F[1,23]=5.02 ; p=0.035$; condition * lateral [left versus right]; Fig. 2D), but this pattern was not related to RT or Activation $(r[24]<0.32 ; p\rangle$ 0.129 ). The global reduction in evoked beta in the MP condition was also present in QL + MP $(F[1,23]=0.01 ; p=0.977$; condition $)$, with an enhanced reduction at midline sites $(F[1,23]=6.17$; $p=0.021$; condition * lateral [lateral versus midline]). While this MP-related reduction in beta occurred as a function of exposure duration, greatest at frontal and posterior sites, there was a trend-level reduction of this temporal change in the QL + MP condition $(\mathrm{F}[1,23]=2.07 ; p=$ 0.164 ; condition * sagittal [frontoparietal versus central] * time; Fig. 1E). The addition of the QL caused a marked global decrease in evoked gamma $(F[1,23]=9.58 ; p=0.005$; condition; see Fig. 3$)$ that was greatest at lateral $(F[1,23]=8.39$; $p=0.008$; condition * lateral [midline versus lateral]) and lateral posterior sites. This reduced the MP-related gamma enhancement at frontal midline and lateral posterior sites $(F[1,23]=6.65 ; p=$ 0.017 ; condition * lateral [midline versus lateral] * sagittal [front versus posterior]; Fig. 1f). The gamma decrease at left hemisphere sites increased over time $(F[1,23]=4.55 ; p=0.044$; condition * lateral [left versus right] * time). None of the gamma patterns were related to RT or Activation $(r[24]<0.25 ; p>0.240)$.

\section{DISCUSSION}

The results of this pilot study suggest that the QL impacts on the neural function effects of the MP in terms of both resting EEG and evoked neural responses to auditory stimuli. With regard to resting EEG, the primary effect was an increase in high frequency activity (gamma) at midline, fronto-posterior sites that was related to a strong enhancement of gamma over the course of the 20-minute condition 


\section{RESTING EEG}
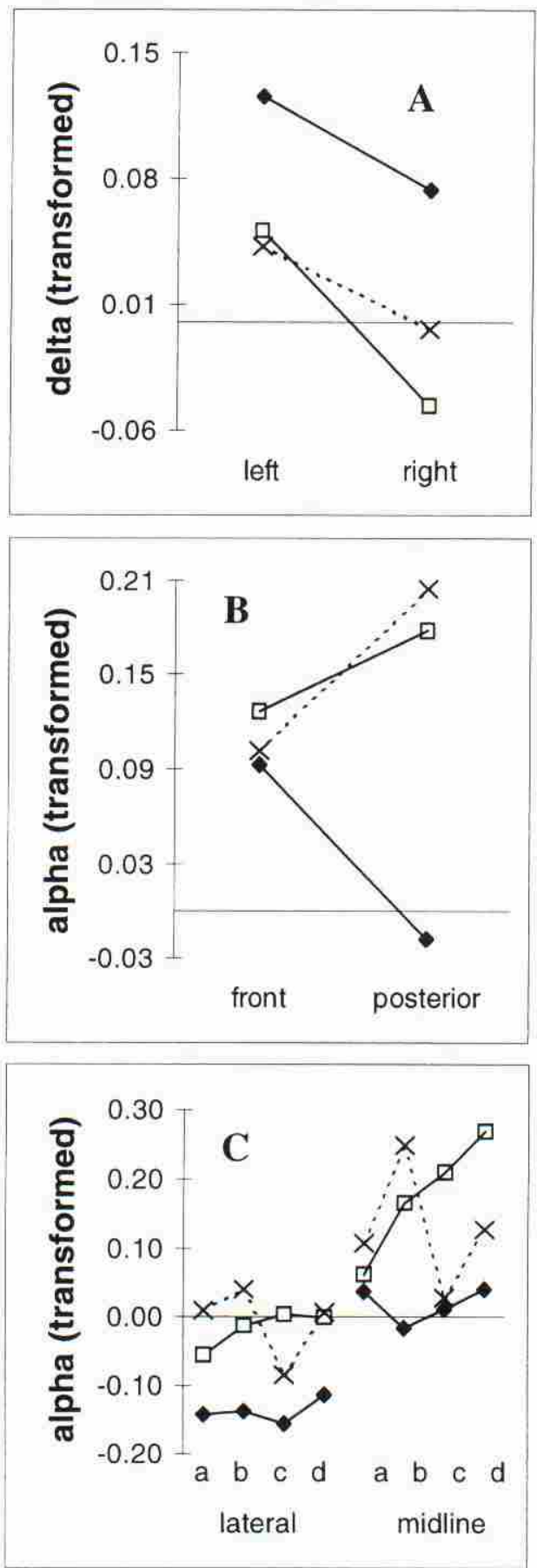

PHASE-LOCKED EEG
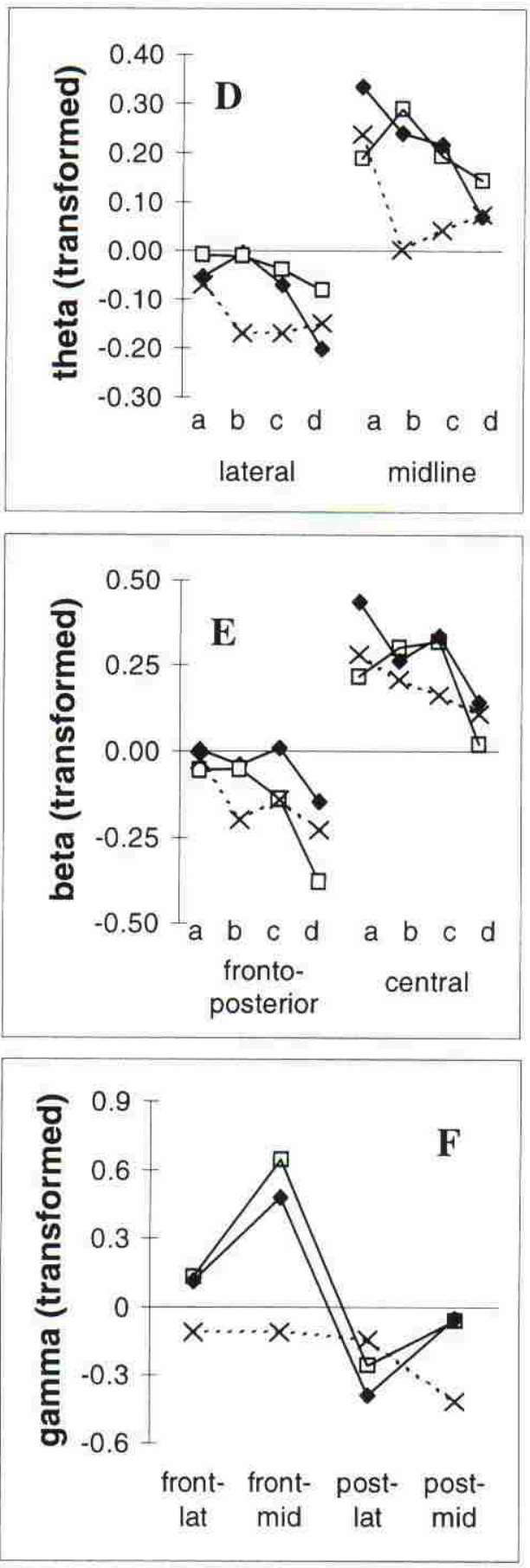

FIG. 1. Effects of the addition of Q Link Ally (QL; Clarus Products International, L.L.C., San Rafael, CA) to mobile phone (MP)-related changes are shown for grand mean resting (EEG) (left column) and phase-locked neural activity (right column). Control results are included as a point of reference and are taken from Croft et al. (2002). CONTROL, filled diamonds; MP, open squares; QL + MP, crosses. A: Delta is reduced at right relative to left hemisphere sites in MP, and this differential is remedied with the addition of QL. B: The MP-related increase in posterior alpha was not affected by the addition of QL. C: The MP-related increase in alpha over time at midline sites is inhibited in the QL + MP condition from time " $\mathrm{c}$ " on. D: No significant differences were found between the MP and QL+MP conditions for evoked theta. E: The MP-related decrease in the evoked beta response was reduced by the addition of QL at fronto-posterior sites. F: Evoked gamma decreased in QL + MP, thus removing the MP-related enhancement at posterior lateral sites (post-lat). 
RESTING EEG
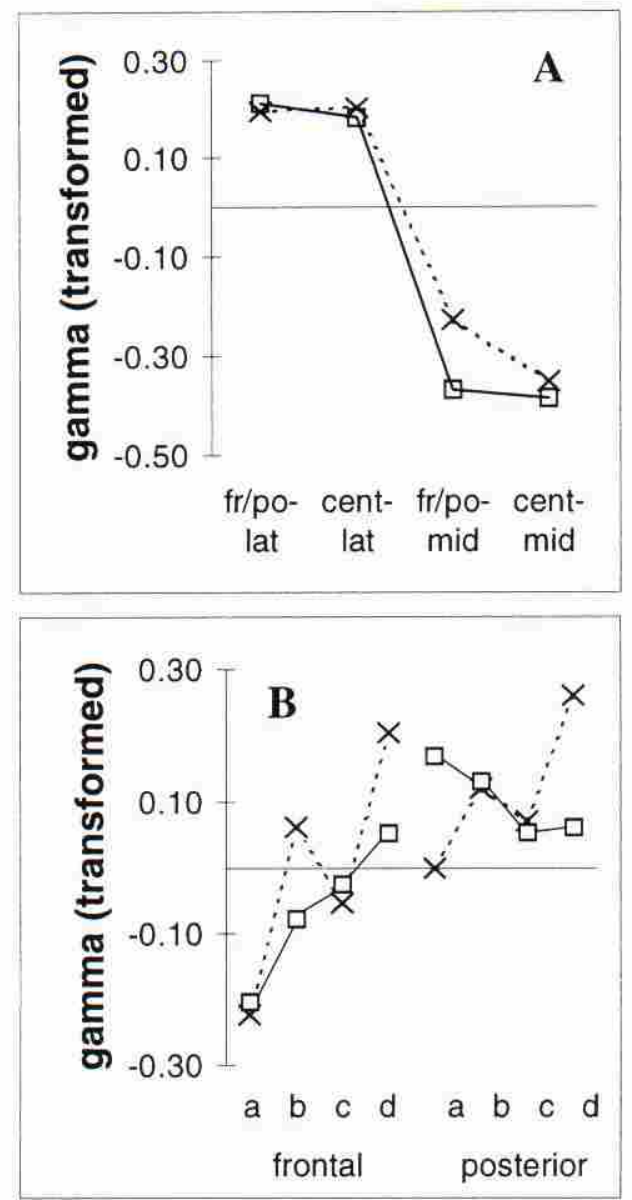

PHASE-LOCKED EEG
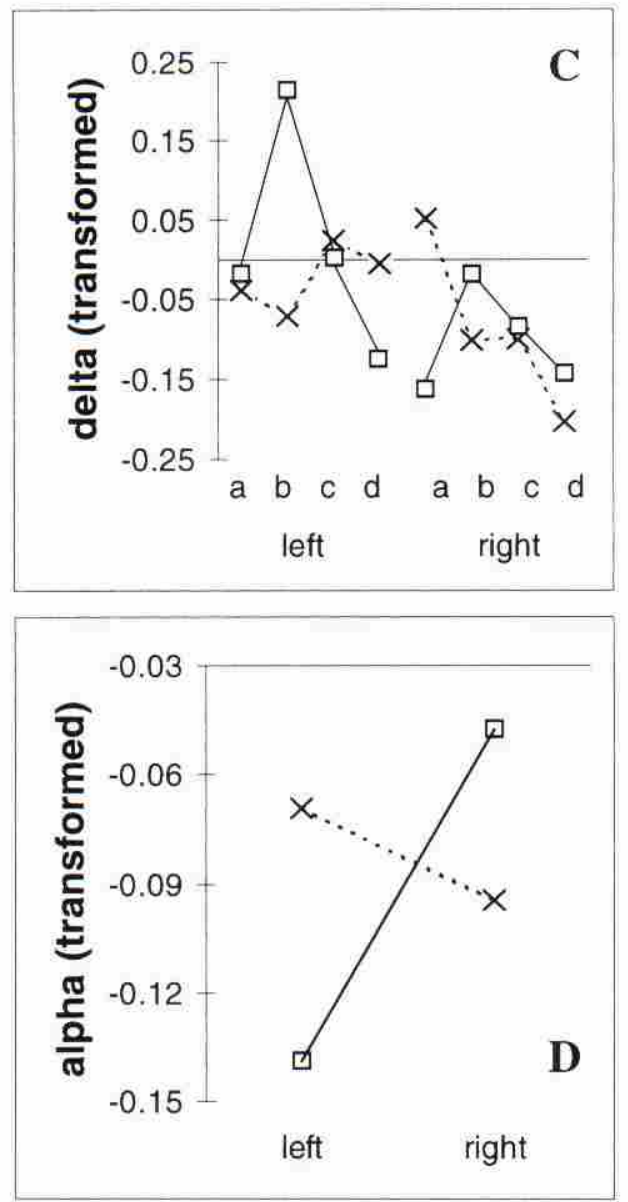

FIG. 2. Effects of the addition of Q Link Ally (QL; Clarus Products International, L.L.C., San Rafael, CA) to the mobile phone (MP) exposure, on resting electroencephalogram (EEG) (left column) and phase-locked neural activity (right column) are shown (grand mean data). MP, open squares; QL + MP, crosses. A: Gamma was enhanced by the addition of QL at fronto-posterior midline sites (fr/po-mid). B: Whereas gamma decreased over time at posterior sites in the MP condition, it increased when the QL was added. C: The change in phase-locked delta over time (decrease at left and relative stability at right hemisphere sites) was reversed by the addition of QL. D: Alpha was enhanced over left and reduced over right hemisphere sites by the addition of QL.

(Fig. 2A,B). The addition of the QL also resulted in trend-level attenuation of the MP-related changes to resting delta and the increase in resting alpha over the MP-exposure period, suggesting that the addition of the $\mathrm{QL}$ does reduce a portion of the MP effect (Fig. 1A,B). Although the attenuation was trend-level only, the conservative nature of the statistical analyses can be seen in Figure 4 where the control and QL group means are indistinguishable. This suggests that where there were significant differences between the control and MP conditions (Croft et al., 2002) but only trend-level differences between the MP and QL + MP condi- tions, this was the result of large variability in the QL + MP condition. There were also alterations to phase-locked neural responses. The addition of the QL caused a right-hemisphere decrease and left-hemisphere increase in the delta attenuation that occurred as a function of exposure duration, a laterality reversal in the alpha band, and a global decrease in the gamma band response. This gamma response was reduced across the scalp, with the reduction largest at frontal midline sites, the result being an attenuation of the MP-related gamma enhancement.

As there is no consensus regarding the mean- 

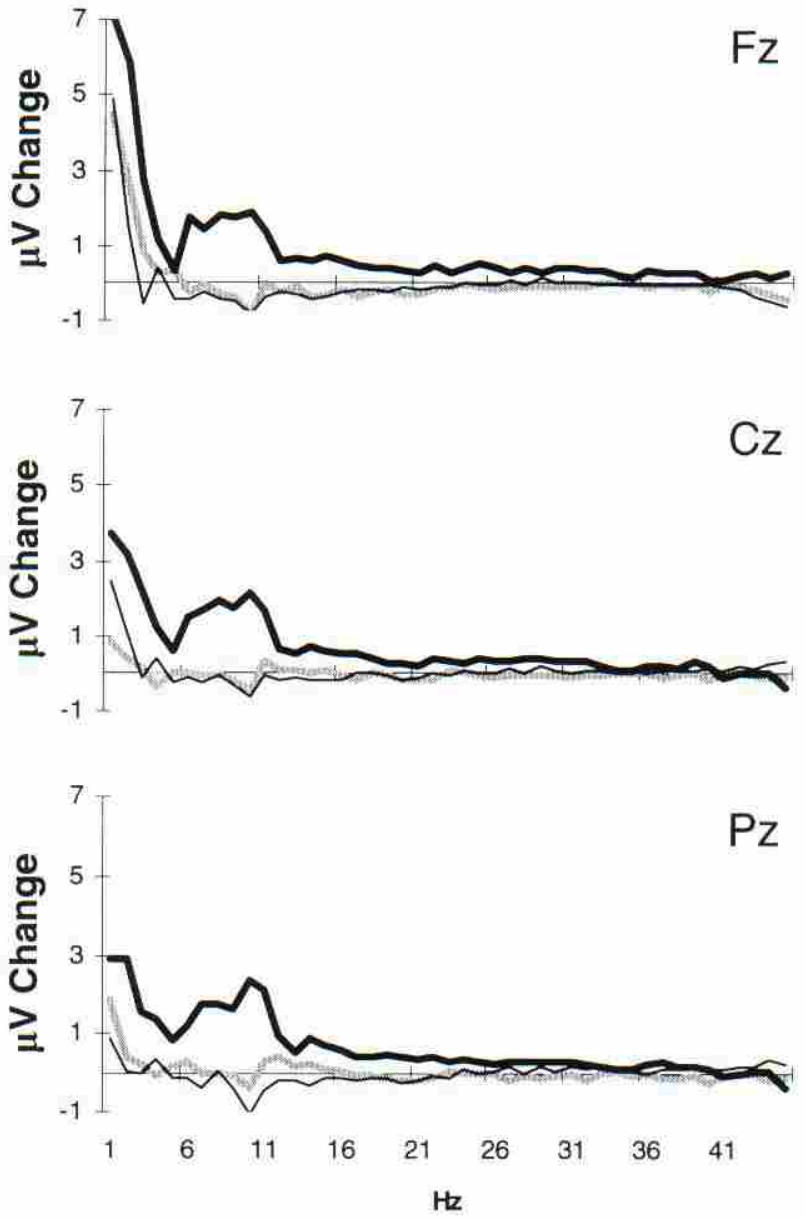

FIG. 3. The grand-mean change over time in resting electroencephalogram (EEG) (2nd minus 1st half of the condition) is shown for the control (thick gray lines), mobile phone (MP) (thick black lines) and Q Link Ally ${ }^{B}$ (QL; Clarus Products International, L.L.C., San Rafael, CA) plus mobile phone (MP) (thin black lines) conditions separately. Of particular interest is that the MP exposure caused a marked increase in alpha $(8-12 \mathrm{~Hz})$ that is not present in QL + MP.

ing of the above resting EEG and neural responses, we are not in a position to fully evaluate the effects. What we may interpret, however, is how the QL-related effects related to the MP-related effects on neural function, and also how these effects related to the behavioral and psychologic indices used. With regard to the effect of the addition of the QL on the MPrelated effects, these were always in the opposite direction to the MP-related effects. That is, the addition of the QL resulted in function that more closely approximated the control condition, suggesting that it was attenuating the effect of the MP. Such attenuation is consistent with the effect of other EMF devices on stressors. For example, it has been reported that low-frequency pulsed electromagnetic fields have protective effects on myocardial infarcts (Albertini et al., 1999; Di Carlo et al., 1999) and hypoxia (Di Carlo et al., 2000), and more closely paralleling the present study, it has been reported that the bioeffects of microwaves are mitigated by the addition of low-frequency noise (Litovitz et al., 1997). With regard to the relation between QL-related changes and both psychologic activation levels and performance, no unequivocal relations were observed. However, trend-level relations were found. Specifically, the QL-related resting gamma increase was related to decreased activation, the QL-related attenuation of MP-related resting alpha increases related to increased activation, and the QL-related lateralization of evoked delta was related to faster reaction times. As these relations were only trend-level it is difficult to draw too much from them, however, they do suggest that as with the effect of MPs themselves (Croft et al., 2002), the addition of the QL resulted in multidimensional effects.

Although it has been demonstrated that the addition of the QL had an effect on neural function (and a trend-level attenuating effect on some of the MP-related changes to neural function), it has not shown which aspect of the QL has caused these effects. That is, although the QL is thought to exert its influence via the interaction of subatomic activity and the biofield,

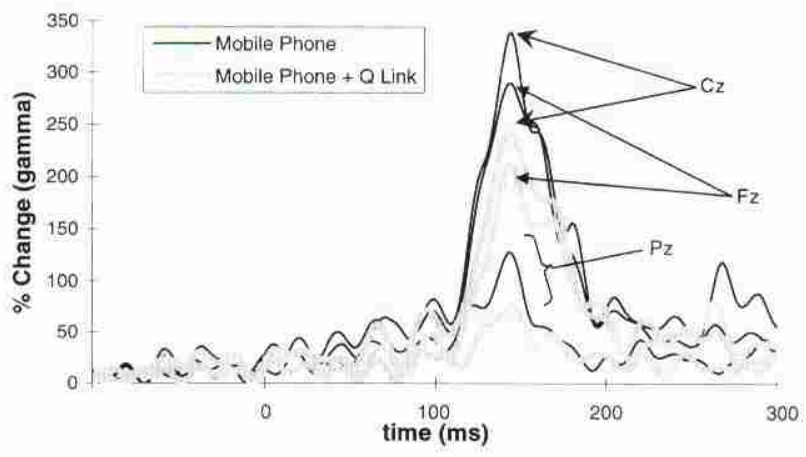

FIG. 4. The grand-mean phase-locked gamma responses are shown as a percentage of baseline at $\mathrm{Fz}, \mathrm{Cz}$ and $\mathrm{Pz}$, for the mobile phone (MP) and Q Link Ally (QL; Clarus Products International, L.L.C., San Rafael, CA) plus mobile phone (MP) (QL + MP) conditions separately. 
what the study has shown is that the addition of the whole QL mechanism (including D.C. and mains frequency as well as Sympathetic Resonance Technology (SRT, ${ }^{\mathrm{TM}}$; Clarus Products International, L.L.C.) has an effect, and so it is possible that the influence was due to the D.C. or $50-\mathrm{Hz}$ power source. Such an interpretation is consistent with the observation that the higher frequencies of neural activity (closer to $50 \mathrm{~Hz}$ ) were more affected by the addition of the QL (30-45 Hz). An extension of this pilot needs to determine this issue through experimental control (e.g., keeping D.C. and 50 $\mathrm{Hz}$ constant in the two conditions and only manipulating the SRT ${ }^{\mathrm{TM}}$ aspect of the QL), but at present the results should be interpreted as being due to either the power source or the SRT TM technology. However, it should be noted that the QL was not physically close to the electrodes $(>30 \mathrm{~cm})$, the distributions of QL effects were not related to the distance between electrodes and the device (but were similar to those typically attributed to neural activity), the magnitude of $50-\mathrm{Hz}$ leakage from the $9-\mathrm{V}$ transformer was too small to account for the QL effect directly, and further, the QL's affect on gamma increased over the course of the exposure. This argues strongly against the thesis that the effects may have been the result of direct influence of the QL on the electrodes, and for the thesis that the effects were due to the interaction of neural function with the addition of the QL.

The present pilot study suggests that the addition of the QL to an active MP-exposure does have an affect on neural function in that it altered both resting EEG and the phase-locked neural response to auditory stimuli. Whether the response was beneficial could not be determined from the present study, however, as there were trends to reduced MP-related effects in the QL + MP condition, this suggests that it was attenuating the MP-related effects. Further research is required to substantiate and clarify these findings.

\section{ACKNOWLEDGMENT}

This research was funded by Clarus Products International, LLC, San Rafael, CA.

\section{REFERENCES}

Albertini A, Zucchini P, Noera G, Cadossi R, Napoleone $\mathrm{CP}$, Parangeli A. Protective effect of low frequency low energy pulsing electromagnetic fields on acute experimental myocardial infarcts in rats. Bioelectromagnetics 1999:20:372-377.

Croft RJ, Chandler JS, Burgess AP, Barry RJ, Williams JD, Clarke AR. Acute mobile phone operation affects neural function in humaans. Clin Neurophysiol 2002; in press.

Di Carlo AL, Farrell JM, Litovitz TA. Myocardial protection conferred by electromagnetic fields. Circulation 1999:99:813-816.

Di Carlo AL, Mullins JM, Litovitz TA. Thresholds for electromagnetic field-induced hypoxia protection: Evidence for a primary electric field effect. Bioelectrochem Bioenerg 2000;52/1(9-16):16.

Lebedeva NN, Sulimov AV, Sulimova OP, Kotrovskaya TI, Gailus T. Cellular phone electromagnetic field effects on bioelectric activity of human brain. Biomed Eng 2000;28:323-337.

Litovitz TA, Penafiel LM, Farrel JM, Kreuse D, Meister R, Mullins, JR Bioeffects induced by exposure to microwaves are mitigated by superposition of ELF noise. Bioelectromagnetics 1997;18:422-430.

Reiser H, Dimpfel W, Schober F. The influence of electromagnetic fields on human brain activity. Eur J Med Res 1995;1:27-32.

Semlitsch HV, Anderer P, Schuster P, Presslich O. A solution for reliable and valid reduction of ocular artifacts applied to the P300. Psychophysiology 1986;23:695-703.

Tabacknick BG, Fidell LS. Using Multivariate Statistics, 2nd ed. New York: Harper Collins, 1989.

Thaya RE. Activation, deactivation adjective checklist: Measurement of activation through self-report. Psychol Rep 1967;20:663-678.

Address reprint requests to: Rodney J. Croft, Ph.D. Centre for Neuropsychopharmacology Swinburne University of Technology Hawthorn 3122, Australia

E-mail:rcroft@swin.edu.au 
Copyright $@ 2002$ EBSCO Publishing 